\title{
A New Strategy to Compare Inverted Rupture Models Exploiting the Eigenstructure of the Inverse Problem
}

\section{by F. Gallovič and J.-P. Ampuero}

Online Material: List of inverted models with explanation of the applied inversion techniques, description of conversion of inverted models to a unified discretization, and figures of original and processed inversion results.

\section{INTRODUCTION}

Finite-fault-slip inversions provide crucial information on earthquake rupture phenomena. Many slip-inversion methods exist and differ in how the rupture model is parameterized and which regularizations or constraints are applied (e.g., Ide, 2007, and references therein). Some methods are utilized even routinely for large earthquakes and published online (e.g., the U.S. Geological Survey website http://earthquake.usgs.gov/, last accessed August 2015). However, the slip-inversion results obtained by various authors for the same event may differ (e.g., Clévédé et al., 2004). There is currently no consensus about which slip-inversion method is preferable, and there are concerns about the reliability of the inferred source models due to the nonuniqueness or ill conditioning of the inverse problem (Hartzell et al., 2007; Zahradník and Gallovič, 2010; Gallovič and Zahradník, 2011; Shao and Ji, 2012). Therefore, slip inversion is still a subject of active research.

A requisite to understand the variability of slip-inversion results across different methods is the characterization of their similarities and differences. Methods to compare spatial distributions of final slip have been previously developed and applied to synthetic and real cases (Clévédé et al., 2004; Razafindrakoto et al., 2015; Zhang et al., 2015). Here, we propose an approach to compare the complete space-time evolution of rupture models.

The basic ideas behind our comparison technique are as follows. If the fault geometry is assumed, the forward problem of the slip inversion is a linear mapping from the model space (the spatial-temporal distribution of slip) to the data space (the seismograms) by means of the representation theorem (e.g., Aki and Richards, 2002). The spectral decomposition of the forward operator and its discrete counterpart, the singular value decomposition (SVD), provide a natural set of basis functions (singular vectors) in the model space. Any source model then can be decomposed into two parts made of linear combinations of singular vectors lying in the coimage and in the null space, which are associated with large and small (or even zero) singular values, respectively. Because only the coimage components provide significant signal, any slip-inversion method should resolve them correctly. The inversion results obtained by different methods may then differ in their null-space contributions, which are implicitly determined by the particular choices of source model parameterization and regularization of each method.

In the subsequent section, we introduce the principles of our comparison method, including an objective method to determine the boundary between the coimage and null spaces. We then briefly describe the Source Inversion Validation (SIV2a) benchmark problem conducted under the SIV (http:// equake-rc.info/SIV/, last accessed August 2015) project. We use the SIV2a setup to illustrate the practical application of our method and compare SIV2a-inversion results obtained by various authors. We also assess how the conditioning of the slip inversion is affected by frequency band, station weighting, network coverage, and crustal model. Finally, we discuss the origin of slip-inversion bias and the implications of our results for the comparison of performance of source-inversion methods.

\section{METHOD}

\section{Background of SVD}

The forward modeling of the ground motions due to slip along a fault of known geometry is a linear mapping from the model space to the data space by means of the representation theorem (Aki and Richards, 2002),

$u_{i}(\mathbf{r}, t)=\iint G_{i j}(\mathbf{r}, \boldsymbol{\xi}, t-\tau) \Delta v_{j}(\boldsymbol{\xi}, t) d \xi d \tau$,

in which $u_{i}(\mathbf{r}, t)$ is the $i$ th component of the ground displacement at position $\mathbf{r}$ and time $t, \Delta v_{j}(\boldsymbol{\xi}, t)$ is the jth component of the slip rate at position $\boldsymbol{\xi}$ on the fault and at time $t$, and $G_{i j}(\mathbf{r}, \boldsymbol{\xi}, t)$ is the ground displacement in the direction $i$ at location $\mathbf{r}$ due to sudden, unit slip in direction $j$ at position $\boldsymbol{\xi}$. In the following, we refer to $G_{i j}$ as the Green's function (GF). 
Detailed presentations of the role of the SVD in understanding the properties of linear-inverse problems can be found in textbooks on geophysical inverse problems (Menke, 2012), or, for example, Olson and Apsel (1982) and Gallovič and Zahradník (2011) in the context of the slip inversions. The SVD approach implicitly assumes as the objective function the L2 norm of data residuals relative to model predictions. Weights can be included to emphasize or depreciate certain aspects of the data (stations, seismic phases, frequency ranges, etc.; note the choice between velocity and displacement data is equivalent to a choice of frequency-dependent weights) or to account for data and model prediction uncertainties (Duputel et al., 2014). Weights generally affect the eigenstructure of the problem as shown later. Approaches to objectively determine these weights are under development (e.g., Duputel et al., 2014) and could be incorporated in our analysis in the future.

We aim to perform a spectral decomposition of the continuum operator in equation (1) by means of a singular value expansion (Hori, 2001; Hansen, 2010). In practice, we achieve this computationally, considering a discretized version of the representation theorem with spatial and temporal sampling dense enough to accurately approximate the singular value expansion of the continuum problem.

After sufficiently fine discretization in time and space, the representation in theorem (1) is written in matrix form,

$\mathbf{d}=\mathbf{G m}$,

in which $\mathbf{d}$ is a data vector of size $N$ that contains the displacements waveforms of all stations and components, $\mathbf{m}$ is a model vector of size $M$ that encompasses all spatial and temporal samples of slip rate, and $\mathbf{G}$ is a design matrix of size $N \times M$ that contains the responses to unit-step slip at each subfault and at each time represented by the elements of $\mathbf{m}$.

The SVD of matrix $\mathbf{G}$ is

$\mathbf{G}=\mathbf{U} \mathbf{\Lambda} \mathbf{V}^{T}$,

in which $T$ represents matrix transpose, $\mathbf{U}$ and $\mathbf{V}$ are orthonormal matrices $\left(\mathbf{U}^{T} \mathbf{U}=\mathbf{I}, \mathbf{V}^{T} \mathbf{V}=\mathbf{I}\right)$ of size $N \times N$ and $M \times M$, respectively, and $\boldsymbol{\Lambda}$ is a diagonal matrix of size $N \times M$ consisting of positive singular values $\lambda_{i}, i=1, \ldots, \min (M, N)$, sorted in descending order. The columns $\mathbf{V}_{(i)}$ of matrix $\mathbf{V}$ are right-singular vectors. They are also eigenvectors of matrix $\mathbf{G}^{T} \mathbf{G}($ size $M \times M)$, forming an orthonormal basis system in the model space and are associated with eigenvalues $\lambda_{i}^{2}$. The columns $\mathbf{U}_{(i)}$ of matrix $\mathbf{U}$ are left-singular vectors, representing orthogonal basis in the data space. The first $\min (M, N)$ vectors $\mathbf{U}_{(i)}$ are projections of model-space basis vectors $\mathbf{V}_{(i)}$ into the data space:

$\mathbf{U}_{(i)}=\mathbf{G V}_{(i)} / \lambda_{i}, \quad i=1, \ldots \min (M, N)$,

that is, normalized seismograms generated by the individual model-space singular vectors.

The generalized solution of the inverse problem is defined as $\tilde{\mathbf{m}}=\mathbf{G}^{\#} \mathbf{d}$, in which $\mathbf{G}^{\#}$ is the pseudoinverse of $\mathbf{G}$ (i.e.,
$\left.\mathbf{G}^{\#}=\mathbf{V} \boldsymbol{\Lambda}^{\#} \mathbf{U}^{T}\right)$, in which $\boldsymbol{\Lambda}^{\#}$ is formed by replacing every nonzero diagonal entry of $\boldsymbol{\Lambda}$ by its reciprocal and transposing the resulting matrix. This is equivalent to expressing $\tilde{\mathbf{m}}$ as a linear combination of basis vectors $\mathbf{V}_{(i)}$,

$\tilde{\mathbf{m}}=\sum_{i=1 \ldots \min (M, N)} \tilde{m}_{i} \mathbf{V}_{(i)}$

in which the $i$ th spectral component of the singular vector expansion of the model is

$\tilde{m}_{i}=\mathbf{U}_{(i)} \cdot \mathbf{d} / \lambda_{i}, \quad i=1, \ldots, \min (M, N)$,

in which the centered dot represents the scalar product. Similarly, the data vector can be expressed as $\mathbf{d}=\sum_{i=1 \ldots N} \tilde{d}_{i} \mathbf{U}_{(i)}$, in which the $i$ th spectral component of the data is $\tilde{d}_{i}=\mathbf{U}_{(i)} \cdot \mathbf{d}$. The first $\min (M, N)$ spectral components of data and model are thus related by $\tilde{d}_{i}=\lambda_{i} \tilde{m}_{i}$. Therefore, the smaller is the singular value, the less sensitive is the data component to a given change of the corresponding model component; in other words, the singular value bears information about the sensitivity of the data to the particular basis function in the model space. Moreover, the relation $\tilde{m}_{i}=\tilde{d}_{i} / \lambda_{i}$ shows that noise in a data component associated with a small $\lambda_{i}$ is amplified by the inversion; the singular value also bears information about the sensitivity of the model to additive data noise. The singular vectors thus can be divided into those lying in either the coimage space or the null space, associated with large and small (or even zero) singular values, respectively.

\section{Comparison Metric}

One can quantify the similarity between an inverted source model $\mathbf{m}$ and a target model by the model variance reduction (MVR), defined as

$\operatorname{MVR}=1-\|\mathbf{m}-\overline{\mathbf{m}}\| /\|\overline{\mathbf{m}}\|$,

in which $\|\cdot\|$ denotes the L2 norm. This metric compares the complete rupture evolutions because $\mathbf{m}$ and $\overline{\mathbf{m}}$ contain samples of the spatial-temporal slip-rate distributions. It is based on the full spectrum of the target and inverted models (i.e., includes contributions from all the singular vectors of $\mathbf{G}$ ). Here we propose to compare the models after expanding them in terms of the (approximate) singular vectors of the continuum problem, then truncating their expansion at a common cutoff singular value $\lambda_{\mathrm{c}}$ sufficiently large to contain only components that, in principle, can be well resolved. For any model $\mathbf{m}$, we define a truncated model $\mathbf{m}_{T}$ by

$\mathbf{m}_{T}=\Sigma_{i=1 \ldots K}\left(\mathbf{V}_{(i)} \cdot \mathbf{m}\right) \mathbf{V}_{(i)}$,

in which $K$ is the index of the smallest singular value larger than $\lambda_{c}$ (Jackson, 1972; Wiggins, 1972; Gallovič and Zahradník, 2011). After truncating both the inverted and the target model, we evaluate the MVR using the following variant of 
equation (7), which we refer to as the truncated model variance reduction (TMVR):

$\mathrm{TMVR}=1-\left\|\mathbf{m}_{T}-\overline{\mathbf{m}}_{T}\right\| /\left\|\overline{\mathbf{m}}_{T}\right\|$.

The choice of $\lambda_{c}$ is crucial. Here it is defined by a departure from the discrete Picard condition, a fundamental condition for stability of regularized solutions of the inverse problem, which requires the spectral amplitudes of the data $\tilde{d}_{i}$ to decay faster than the singular values $\lambda_{i}$ (Hansen, 1990, 2010; Visser, 2004). In particular, the Picard condition ensures that a truncated SVD solution $\left(\sum_{i=1 \ldots K} \tilde{d}_{i} / \lambda_{i} \mathbf{V}_{(i)}\right)$ converges as more eigenvalues are included (as $K$ is increased). As illustrated later (Fig. 2b), inaccuracies in GFs due to uncertainties in the velocity model or fault geometry cause $\tilde{d}_{i}$ to stagnate or even increase at small $\lambda_{i}$. This departure from the decay behavior of the data spectrum is usually clear enough to provide an objective choice of cutoff for truncated SVD solutions (Hansen, 1990; Visser, 2004). Here, we use that same rationale to set the cutoff singular value $\lambda_{\mathrm{c}}$ for our TMVR comparison metric.

\section{TEST CASE: SIV2A MODEL}

Here we present the main features of the SIV2a benchmark problem. A complete description can be found at the SIV web portal (http://equake-rc.info/SIV, last accessed August 2015). A complex kinematic rupture model with oblique mechanism on a dipping fault plane is considered. The fault is $40 \mathrm{~km}$ long (along strike) and $20 \mathrm{~km}$ wide (along dip), and the seismic moment is $3.5 \times 10^{19} \mathrm{~N} \cdot \mathrm{m}\left(M_{\mathrm{w}} 7.0\right)$. The source model is kinematic and adopts the regularized Yoffe slip-rate function (Tinti et al., 2005) with variable rise time and rupture speed along the fault. The rake and dip angles are constant, but only approximate value ranges are provided $\left(230^{\circ}-250^{\circ}\right.$ and $40^{\circ}-50^{\circ}$, respectively). The hypocentral depth is also given only approximately, ranging between 9 and $11 \mathrm{~km}$. Here we adopt the middle values of the provided intervals: dip $45^{\circ}$, rake $240^{\circ}$, and depth $10 \mathrm{~km}$.

The SIV2a rupture model (hereafter referred to as the target model) was initially unknown to the participants of the source-inversion blind test but is presently available upon request. Figure 1a shows the spatial distribution of slip of the target model and slip-rate snapshots showing the temporal evolution of the rupture. The model was originally provided at a fine spatial-temporal discretization. (E) We downsampled it as described in the electronic supplement to this article, at a sampling rate sufficient for the maximum frequency we considered (see below).

The fault is embedded in a layered isotropic velocitydensity structure, the parameters of which were given to the modelers. Synthetic waveforms evaluated at 40 receivers distributed from above the fault to approximately $30 \mathrm{~km}$ distance to the fault (see Fig. 1b) were available as data for the source inversion.

We calculate GFs with the Axitra code (Coutant, 1989) based on the discrete wavenumber method by Bouchon (1981). The waveforms are band-pass filtered between 0.05 and $0.5 \mathrm{~Hz}$ using a causal four-pole Butterworth filter. These GFs are not those used to compute the disseminated benchmark waveform data. Indeed, the variance reduction (VR) between our synthetics for the target model and the benchmark data is $\mathrm{VR}=0.95$. This minor mismatch can be attributed to the use of slightly different fault-plane position and orientation, a rake angle, or to minor numerical inaccuracies and different choices of algorithmic parameters in wave propagation codes. Similar differences can be expected for the other benchmark participants.

\section{RESULTS}

Figure 1c shows the spectrum of the design matrix (i.e., the distribution of its singular values). The spectrum decays approximately exponentially with the increasing index. This decay is steady, without sharp break, and hence there is no obvious definition of the boundary between coimage and null spaces. We propose to define an effective boundary, that is, a cutoff singular value $\lambda_{\mathrm{c}}$ that separates resolved from unresolved source features, using the discrete Picard condition.

\section{SIV2a Test Case with Precise GFs}

We perform the first tests on the inversion results obtained with the method of Gallovič et al. (2015), a linear-inversion method with multitime window parameterization, unconstrained rupture speed and rise time, constrained total rupture duration (12 s or less), and two regularization constraints: a $k^{-2}$ slip covariance function ( $k$ being the wavenumber) and positivity of slip rate (see also Sokos et al., 2015). The degree of smoothing is controlled by a parameter $\sigma_{D}$ defined by Gallovič et al. (2015). A value of $\sigma_{D}=0.1 \mathrm{~m}$ is adequate for realdata applications with imperfect GFs, but in this controlled experiment we use weaker smoothing with $\sigma_{D}=0.01 \mathrm{~m}$. Exact GFs are used in this test: we apply the inversion procedure to data evaluated using our own version of the target model and our own computed GFs (the disseminated waveforms are not used at this point).

The resulting source model has MVR $=0.75$ (without truncation). Its TMVR decreases toward the MVR value as a function of decreasing $\lambda_{c}$ (Fig. 2a). It is close to 1 when $\lambda_{\mathrm{c}}>\lambda_{\max } / 20$, in which $\lambda_{\max }$ is the largest singular value. The spectral amplitudes of the data $\tilde{d}_{i}$ (red curve in Fig. $2 \mathrm{~b}$ ) decay slightly faster than the singular values (black line), which shows that the discrete Picard condition is satisfied.

\section{SIV2a Test Case with Imprecise GFs}

We now consider a case in which the GFs used in the forward and inverse modeling are not identical: we invert the disseminated benchmark data using the method of Gallovič et al. (2015) based on our GFs. The obtained model is denoted as Gallovic0.01 (see (E) Table S1). Its final slip and slip-rate snapshots are shown in Figure 3 (left-most column). Its MVR is 0.66, lower than in the case of precise GFs. Its TMVR (Fig. 2a) decreases as a function of decreasing $\lambda_{c}$, from relatively large values $\sim 0.95$ to 0.66 , with faster decay at $\lambda_{\mathrm{c}}<1 / 10 \sim 1 / 20 \lambda_{\max }$. The 
(a)
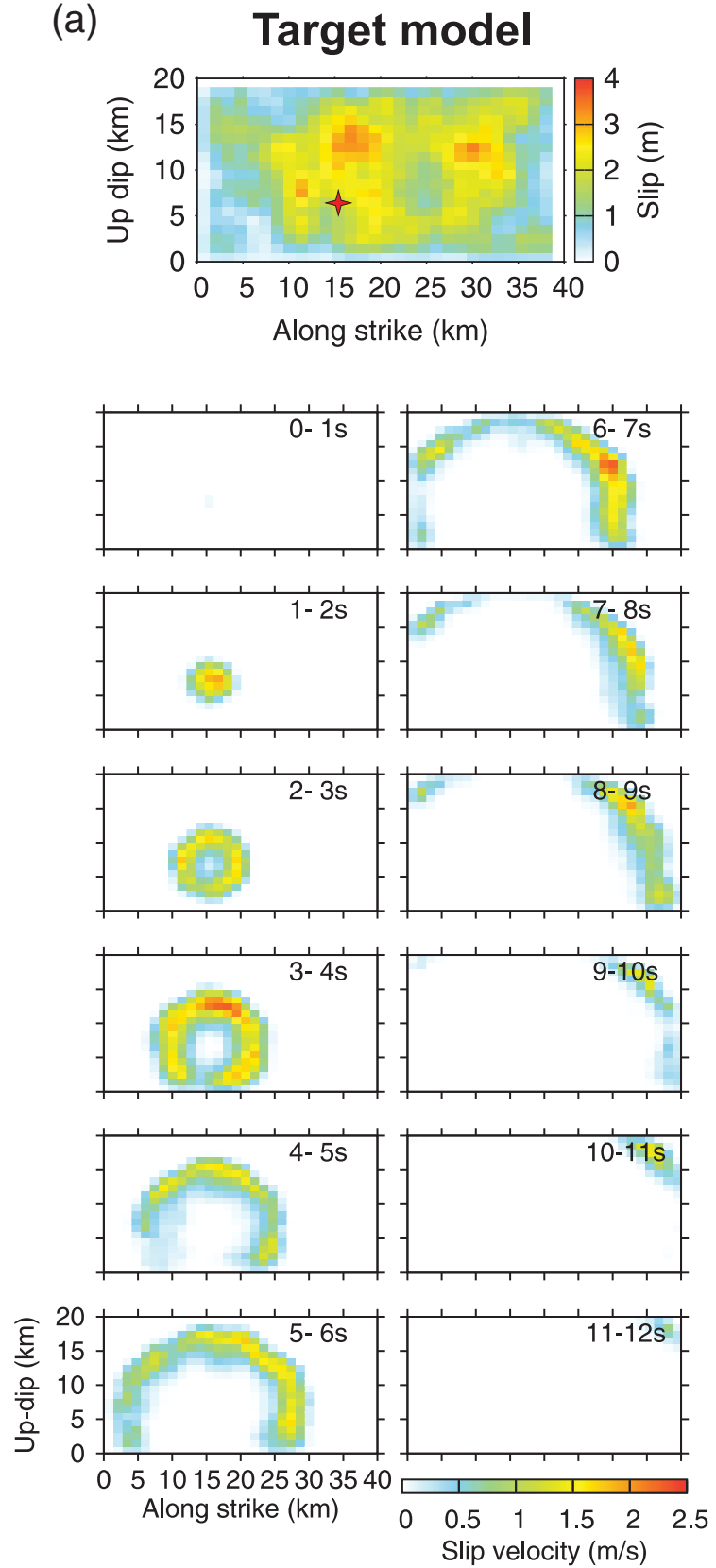

(b)
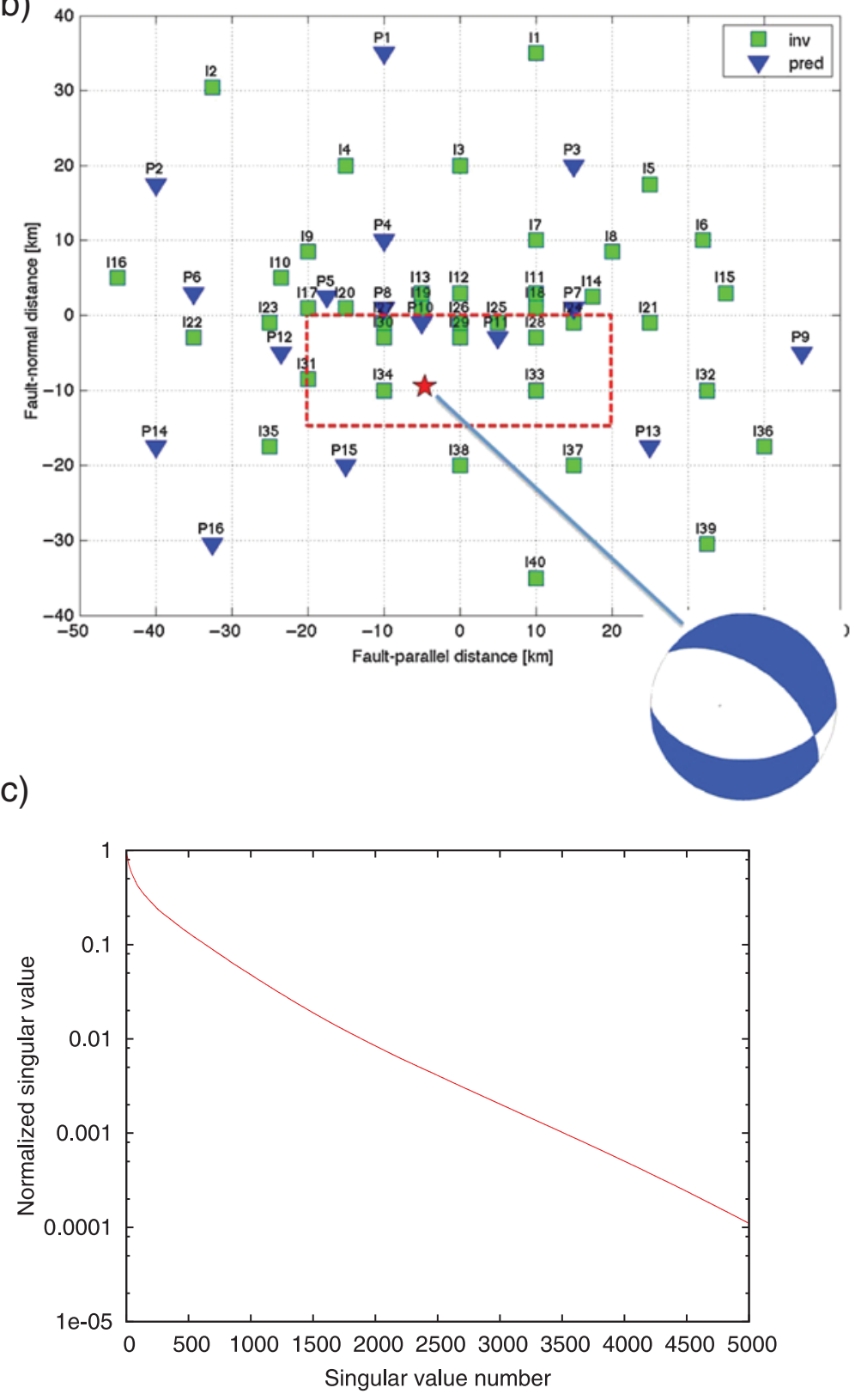

- Figure 1. Properties of the Source Inversion Validation (SIV2a) benchmark. (a) (top-most panel) Slip distribution and (bottom panels) slip-rate snapshots of the target rupture model. (b) Distribution of stations, surface projection of the fault (red rectangle), and the source mechanism (focal mechanism plot). (c) Normalized singular values of the $\mathbf{G}$ matrix. Because the discretization is very fine relative to the considered frequency range, the spectrum very closely approximates the spectrum of the continuum forward operator.

spectral amplitudes of the data (green in Fig. 2b) decay as a function of decreasing $\lambda_{i}$ like those obtained with perfect GFs when $\lambda_{i}>1 / 10 \sim 1 / 20 \lambda_{\max }$, but stagnate at smaller $\lambda_{i}$. This clear transition of the decay behavior of the data spectrum is caused by the use of imprecise GFs in the inversion and signals a departure from the discrete Picard condition. This suggests that, in the present case, a reasonable choice for the cutoff in model comparisons based on TMVR is $\lambda_{\mathrm{c}} \sim 1 / 10 \lambda_{\max }$. This level corresponds to a coimage subspace dimension of 637 . This number does not depend on the discretization, provided it is fine enough to accurately approximate the continuum formulation of the problem.

\section{Comparison of Inverted Models from the SIV2a Solution Database}

Several other modelers have made their solutions of the SIV2a benchmark available. (E) Table S1 lists the model names and provides brief descriptions of their inversion methods and data preprocessing. The methods include the linear-inversion method by Sekiguchi et al. (2000) (Asano model provided by 

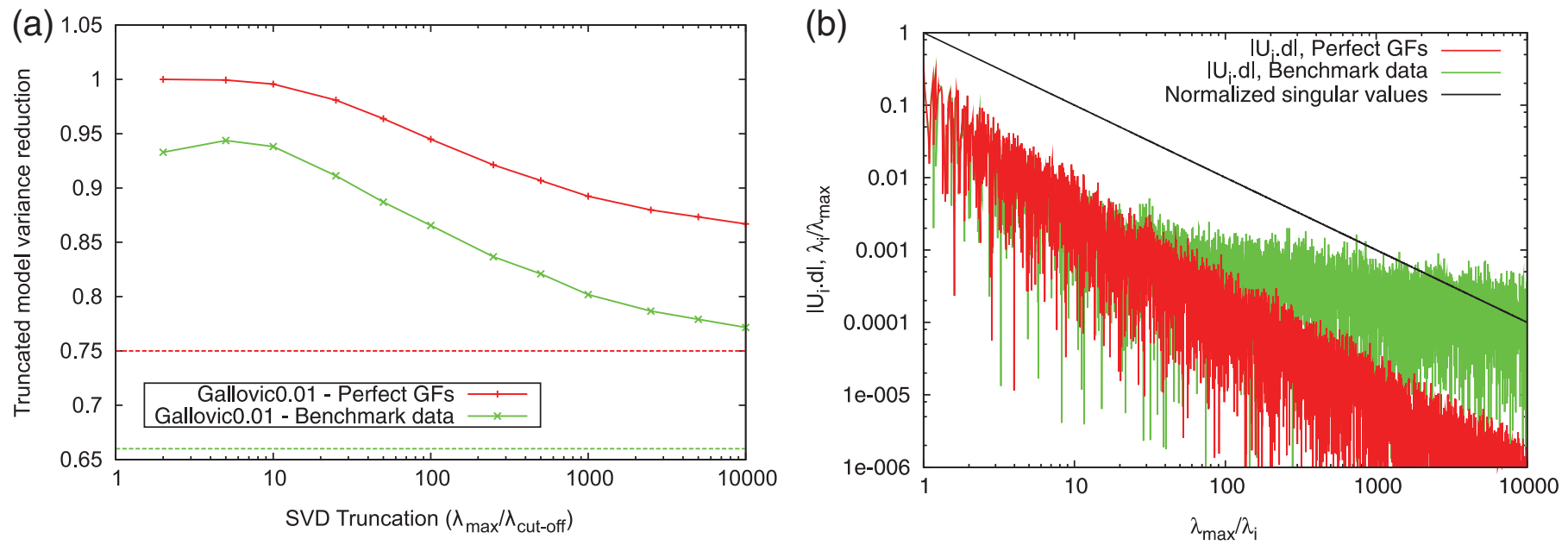

- Figure 2. Role of the precision of the Green's functions (GFs) on the inversion results for the SIV2a benchmark. (a) Model variance reduction (MVR) between the truncated true model and the truncated solution Gallovic 0.01 as a function of the cutoff singular value (solid lines) when perfect (red line) or imprecise (green line) GFs are considered. MVRs between the inverted and true models without truncation are indicated by dashed lines (same color scheme). (b) Spectral components of the data for perfect (red) and imperfect (green) GFs as a function of (inverse) singular values. A decay faster than the black solid line indicates satisfaction of the discrete Picard condition for stability of regularized solutions of the inverse problem.

K. Asano see Asano and Iwata, 2011), a nonlinear formulation by Razafindrakoto and Mai (2014) (Hoby model provided by $\mathrm{H}$. Razafindrakoto), and the method by Twardzik et al. (2012) based on a simplified source parameterization comprising two elliptical subfaults with constant rupture velocities (model CedricT3 provided by C. Twardzik).

(E) We resampled these solutions to a common discretization as described in the electronic supplement. Figure 3
Gallovic0.01
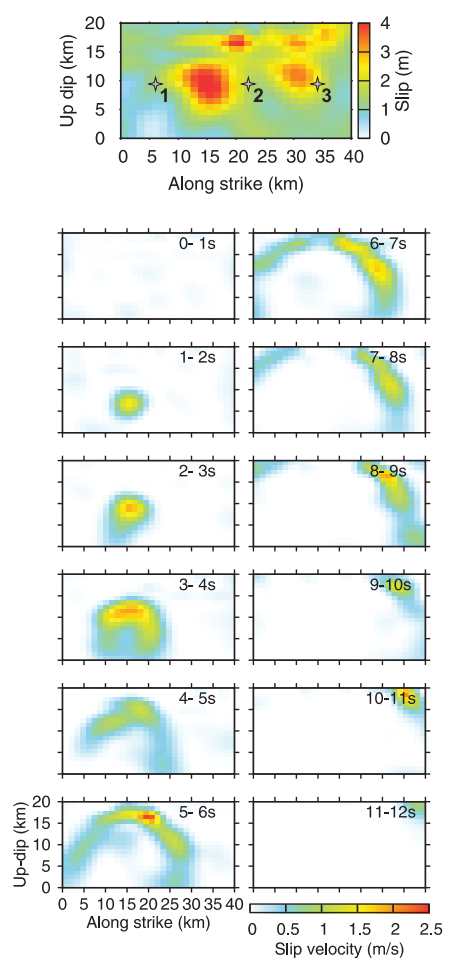

Hoby
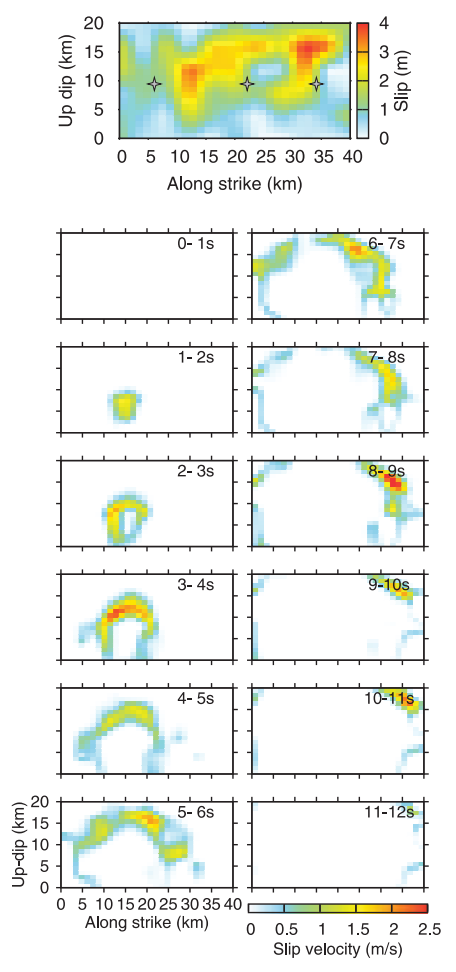

CedricT3
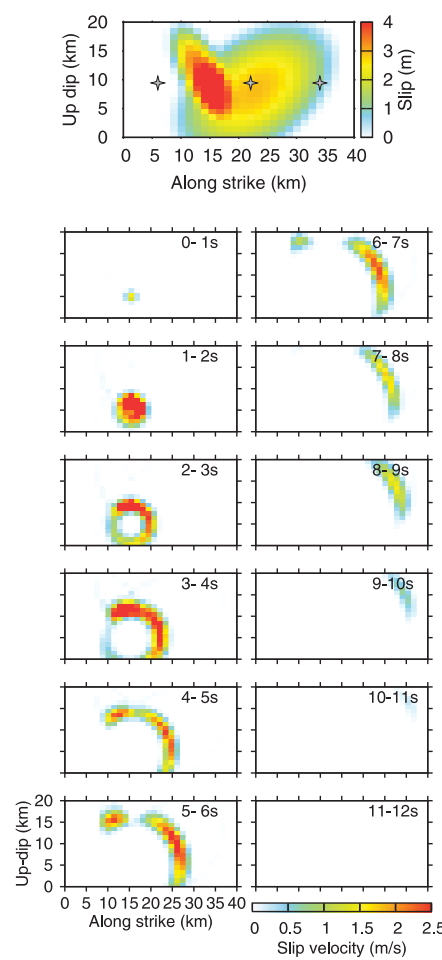

Asano
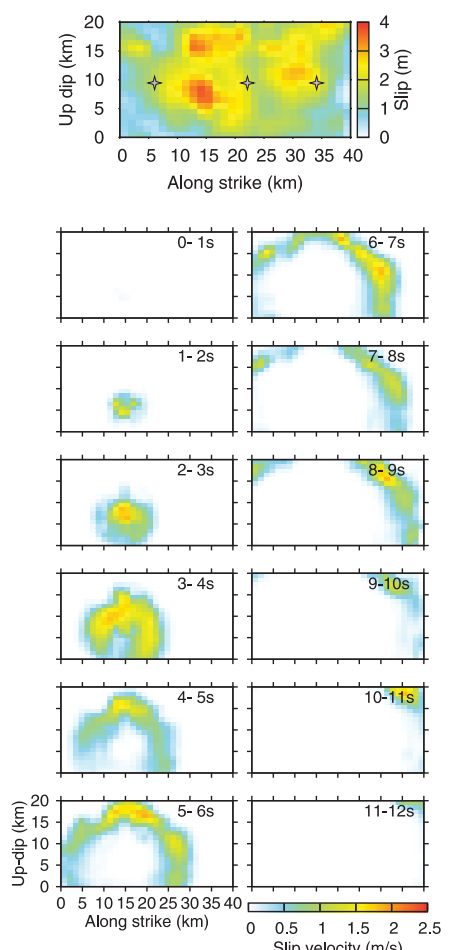

- Figure 3. Selected inversion results of the SIV2a benchmark obtained by various authors (see () Table S1): slip distributions (top-most panels) and slip-rate snapshots (bottom panels). Stars in the slip plots denote locations for which slip rates are shown in Figure 4a. (E) More models are shown in the electronic supplement. 

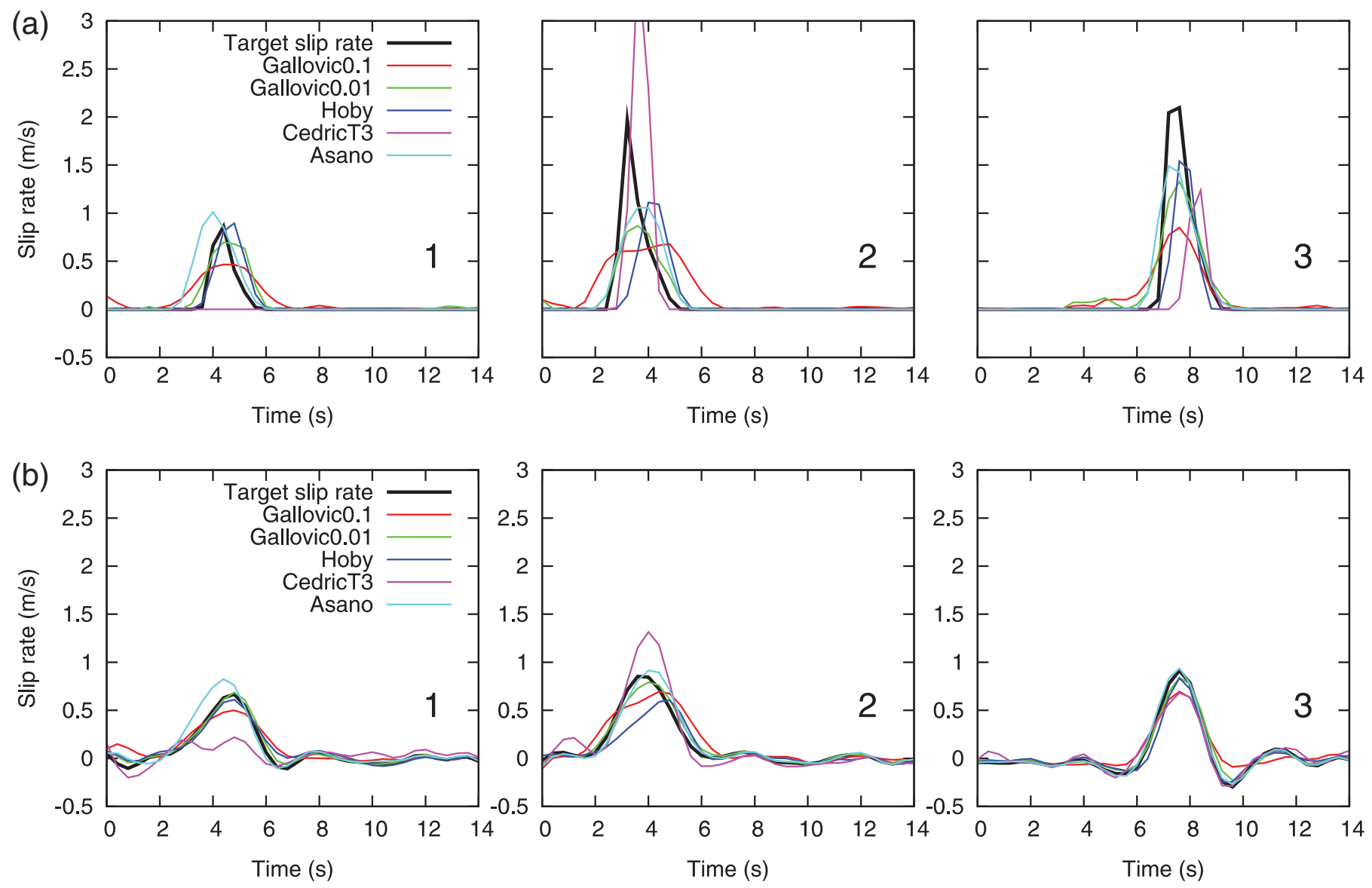

$\Delta$ Figure 4. (a) The comparison of slip-rate functions from inverted and target models at three selected points on the fault indicated in Figure 3. (b) Same as (a) but after truncation at one-tenth of the largest singular value. After truncation, the slip rates become much more similar to each other.

shows the final slip distribution and slip-rate snapshots for selected models (E) all models are shown in the electronic supplement). These are to be compared with the target model in Figure 1a. Figure 4a shows a comparison of the inferred slip rates at three selected points on the fault depicted in the slip maps of Figure 3. The MVR of each model is listed in Table 1.
All the models resolve well the basic character of the rupture propagation (see the snapshots in Fig. 3). However, at a more detailed level, differences among the methods become evident, as in the slip-rate functions shown in Figure 4a. For example, in the CedricT3 solution, point 1 has zero slip rate, whereas point 2 has an exaggerated peak slip-rate amplitude. Solution Gallovic0.1 (with stronger smoothing than in the

\begin{tabular}{|c|c|c|c|c|}
\hline Data and Model Varianc & the Variou & $\begin{array}{l}1 \\
\text { n Solutions }\end{array}$ & rget Model & /2a Benchn \\
\hline & & & & \\
\hline Model Name & Complete & Truncated & Complete & Truncated \\
\hline Gallovic 0.01 & 0.66 & 0.94 & 0.997 & 0.995 \\
\hline Gallovic0.1 & 0.53 & 0.84 & 0.981 & 0.980 \\
\hline Hoby & 0.50 & 0.83 & 0.825 & 0.824 \\
\hline Cedrict3 & -0.20 & 0.65 & 0.700 & 0.699 \\
\hline Asano & 0.61 & 0.86 & 0.960 & 0.959 \\
\hline Target (with perfect GFs) & - & - & 1.000 & 0.996 \\
\hline
\end{tabular}


Gallovic0.01 model discussed previously) provides broader sliprate functions centered at the true maximum.

Figure 3 also shows a comparison of the final slip maps (top panels). The most dissimilar solution is model CedricT3. This model has a simple parameterization comprising two ellipses (see (E) Table S1) with clearly visible contributions in the retrieved slip map. The ellipse with the largest amount of slip is located around the true hypocenter. Nevertheless, a similar concentration of slip at the hypocenter is common to all the other models, although in the target model the maximum slip is located $\sim 5 \mathrm{~km}$ above the hypocenter. Other details of the slip distribution are also resolved imperfectly; for example, the location of subsurface asperities is biased toward shallower depths. The deficiencies in the recovery of final slip should come as no surprise, given the limited frequency band of the data considered and the inherent nonuniqueness of the inverse problem. In particular, the applied high-pass filter above $0.05 \mathrm{~Hz}$ effectively downweights the static deformation.

We analyze these four models in the same way as for model Gallovic0.01. Figure 5 shows their TMVR as a function of the truncation cutoff $\lambda_{\mathrm{c}}$. Dashed lines indicate their MVR without truncation (see also Table 1). Similarly to the Gallovic0.01 model, most truncated solutions agree well with the truncated target model (TMVR $>0.8$ ) if $\lambda_{\mathrm{c}}>\sim 1 / 10 \lambda_{\max }$. The exception is model CedricT3, although it still has a much higher agreement after truncation (TMVR $=0.6)$ than without it $($ MVR $=-0.2)$.

Figure 6 shows the final slip and slip-rate snapshots of some of the analyzed models truncated at $\lambda_{\mathrm{c}}=1 / 10 \lambda_{\max }$ (for a plot with all the models, (E) see the electronic supplement). There is a striking visual similarity between the truncated models, consistent with the large TMVR values presented above. Figure $4 \mathrm{~b}$ shows slip rates after truncation at three selected points on the fault indicated in Figure 3. The similarity of slip rates among different methods is significantly higher with truncation than without it (Fig. 4a). Even though at point 1, the

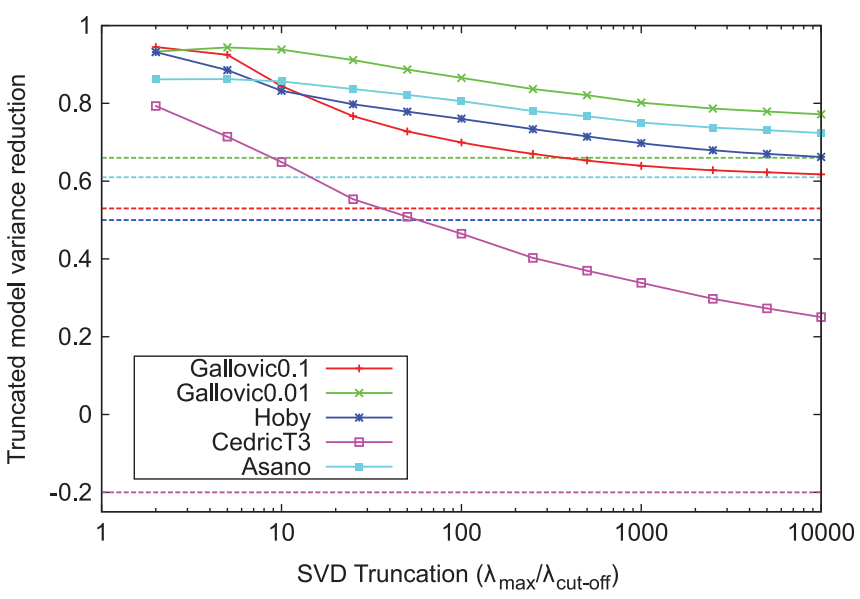

- Figure 5. MVR as a function of the cutoff singular value for all the analyzed models from the SIV database (solid lines). Dashed lines denote MVRs between inverted and true models without truncation.
CedricT3 solution has zero slip rates, after truncation the slip rates become nonzero. Although this model is the least similar to the target model, after truncation the similarity improves significantly. Thus, we conclude that all the inverted models agree within the coimage subspace associated with singular values $>\sim 1 / 10 \lambda_{\max }$. This boundary thus defines the effective range of the problem, which encapsulates the source features that are resolvable, and agrees with the bound indicated by the discrete Picard plot (Fig. 2b).

We also evaluate the contribution from the null-space in terms of the data fit. Table 1 lists the variance reduction in the data space (DVR). There is a very small difference $(\sim 0.1 \%)$ in DVR between the truncated models and those without truncation. This confirms that the contribution of the truncated singular vectors to the signal is very weak. This, in turn, means that the contribution of these singular vectors to the inverse solutions is highly vulnerable to any inconsistency in the fault geometry or velocity model considered.

Finally, we compare the original target model (without truncation) with the truncated one. Both models are shown with the same color scale in the two left-most columns in Figure 6. The truncated model captures well the general rupture propagation characteristics, which is expressed by the relatively high MVR (0.58) between the two models. Nevertheless, the final slip distributions are significantly different (compare the two top left panels in Fig. 6). The slip distribution of the truncated model is biased, having exaggerated slip values in relatively small areas near the projection of the stations on the fault. This is a manifestation of the nonuniform resolution of the inverse problem. In terms of waveform fit, the truncated target model reproduces the original data very well up to $0.5 \mathrm{~Hz}(\mathrm{DVR}=0.996)$.

\section{Factors Controlling the Spectrum of the G Matrix}

The steepness of the decay of the spectrum of the forward operator (in the discrete form represented by the $\mathbf{G}$ matrix) determines the conditioning of the inverse problem, which is defined here as the number of singular values larger than the given cutoff $\lambda_{\mathrm{c}}$. Here we analyze the effect of various features of the slip-inversion setup on the $\mathbf{G}$ matrix spectrum. For this test, we utilize the geometry and parameters of a real earthquake study, the $2009 M_{\mathrm{w}} 6.3$ L'Aquila earthquake (Gallovič et al., 2015), including additional phantom stations denoted as EX_.

As a reference-inversion setup, we assume nine real stations near L'Aquila (see Fig. 7a, excluding the EX_stations), a 1D layered crustal model, maximum frequency $F_{\max }=0.5 \mathrm{~Hz}$, and distance-independent weights. The bold black line in Figure 7b shows the spectrum of singular values of the corresponding $\mathbf{G}$ matrix normalized by their maximum.

We then perform tests, in which the model features are altered one at a time. Colored curves in Figure 7 show the resulting spectra of $\mathbf{G}$. In the first test, we decrease the maximum frequency to $0.3 \mathrm{~Hz}$. This leads to faster decay of the spectrum of $\mathbf{G}$, and thus its conditioning worsens significantly. The reason is that decreasing the maximum frequency decreases the 


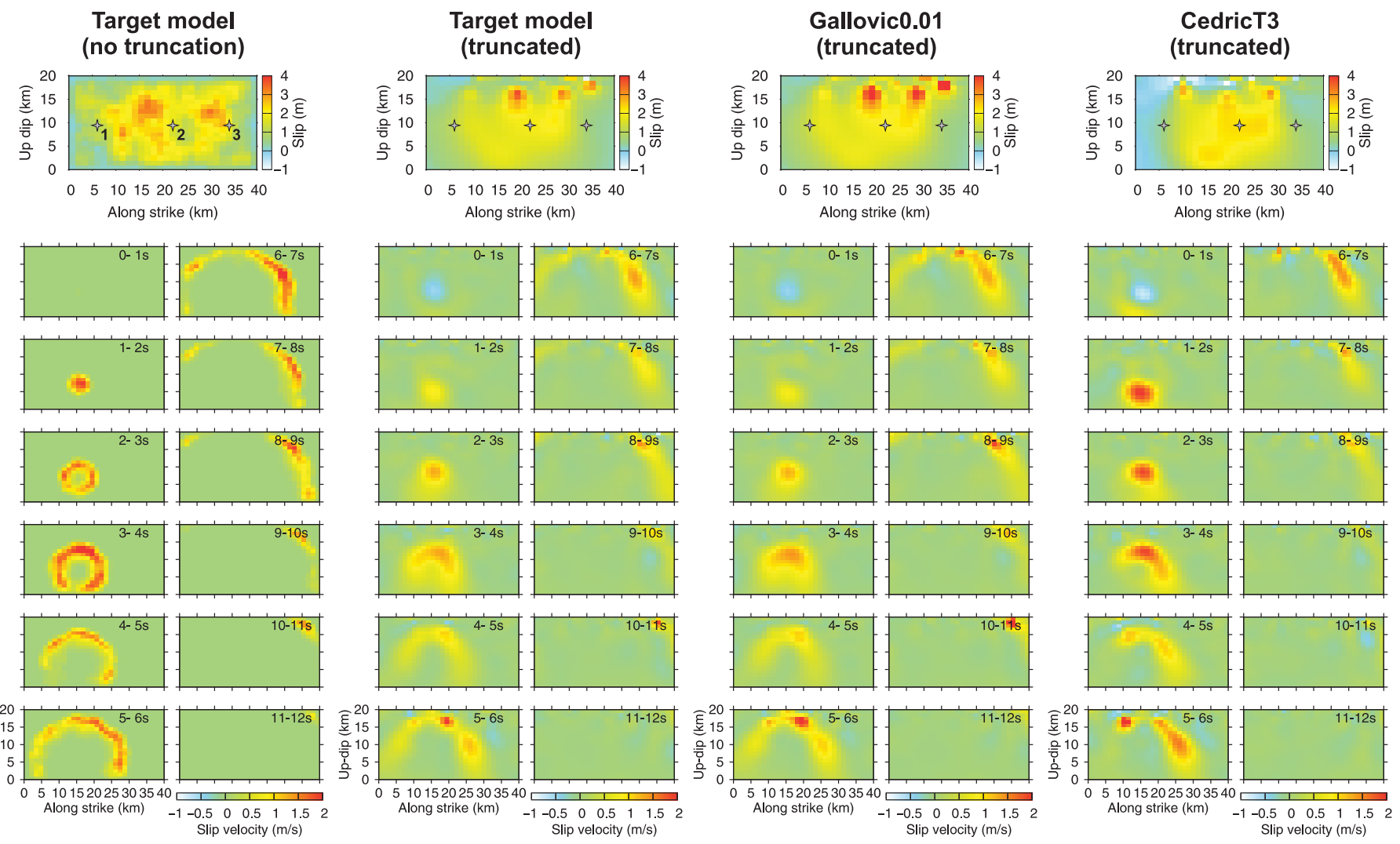

A Figure 6. Target model and two selected inversion results (columns) truncated with cutoff at one-tenth of the largest singular value: slip distributions (top-most panels) and slip-rate snapshots (bottom panels). The original target model is shown on the left, for reference, in the same color scale. Note the very good agreement between the inverted models and the target model after truncation. Stars in the slip plots denote locations for which slip rates are shown in Figure 4a. (E) All the models are shown in the electronic supplement.

(a)

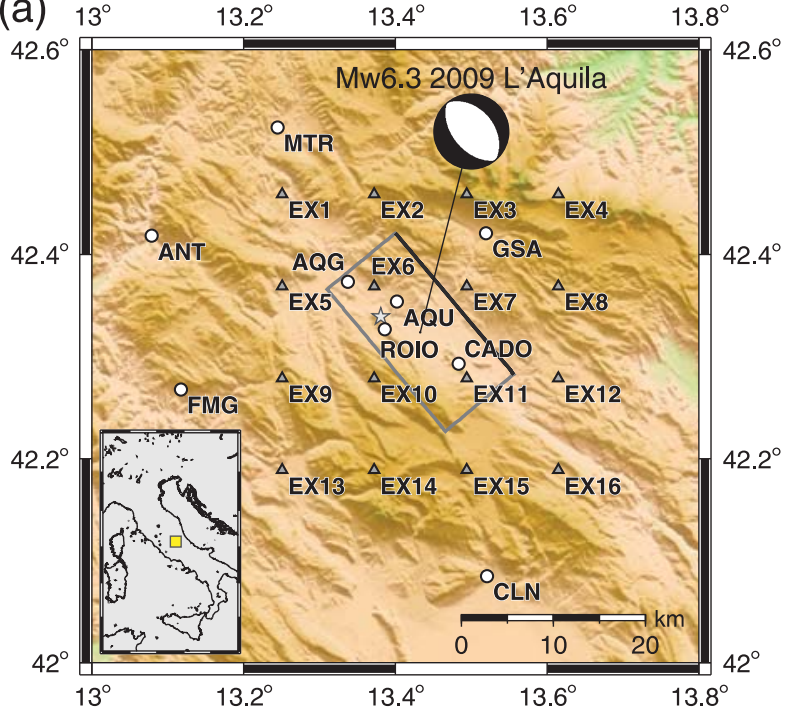

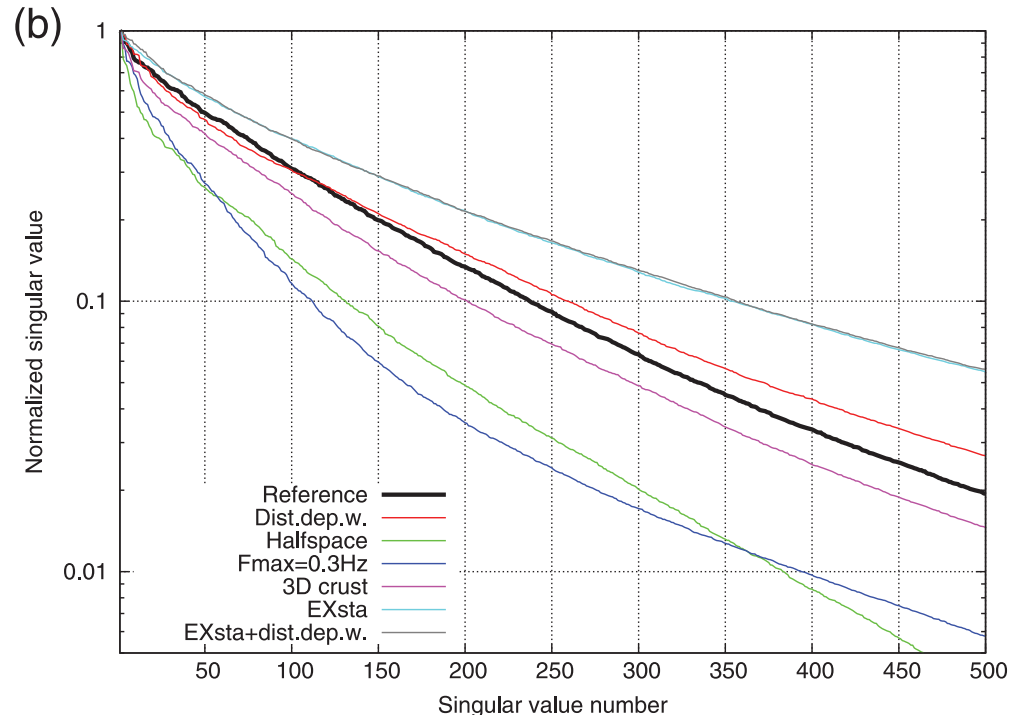

A Figure 7. An assessment of factors controlling the spectrum of the forward operator (i.e., of the $\mathbf{G}$ matrix after discretization). (a) Real (circles) and phantom (triangles, EX_) stations and fault of the L'Aquila earthquake (gray rectangle with the top denoted by the black line); the yellow rectangle in the inset marks the study area. (b) Normalized singular values of the forward $\mathbf{G}$ matrix and their dependence on various features and parameters of the inversion setup (see text for explanation). 
variability of the GFs along the fault and hence their information content, which makes the inversion less constrained.

As a next test, we apply distance-dependent weights equal to $\max (4 R / L, 1)$, in which $L$ is the fault length and $R$ is the Joyner-Boore fault distance. This weighting scheme strengthens the role of the distant stations, and the conditioning of G improves, although its effect is less significant than that of changing the frequency range (Fig. $7 \mathrm{~b}$ ). We then add stations to the inversion (denoted as EX_in Fig. 7a). Figure $7 \mathrm{~b}$ shows how the conditioning of $\mathbf{G}$ improves. The figure also demonstrates that there is not much difference whether the distancedependent weights are considered or not.

We further test the effect of the crustal model on the inversion. To avoid confusion, we note that this analysis does not depend on any data, so we do not analyze effects of imprecise GFs here. We consider two additional crustal models: a halfspace and a smooth 3D tomographic velocity model by Di Stefano et al. (2011). Details on the velocity model and the GF calculations are provided by Gallovič et al. (2015). In both cases, the conditioning of $\mathbf{G}$ worsens, but it is more severe in the former case. We attribute this to the decreased variability of GFs along the fault due to the smoothness of these two models and hence to the decrease of the wavefield information content. In contrast, the layered crustal model contains strong velocity interfaces that make the wave propagation more complex (reflected and transmitted waves, multiples, dispersion of surface waves, etc.).

The behavior of the conditioning of $\mathbf{G}$ presented here must be considered only qualitatively. The exact behavior is case dependent, and the presented results are only intended to provide general trends. Moreover, the changes of the inversion setup not only change the spectrum of $\mathbf{G}$, but also the shapes of the singular vectors. A synoptic quantification of such changes is difficult, mostly because the singular vectors are $3 \mathrm{D}$, but deserves further study.

\section{DISCUSSION}

\section{Effect of Station Distribution on Final Slip Bias}

Although the inverted models explain $99 \%$ of the data and reproduce the general space-time characteristics of rupture propagation of the true source reasonably well after projection on the effective coimage subspace, their final slip distribution is significantly biased, consisting of a few peaks below the stations overlying the rupture area (see Fig. 6). This nonuniform resolution (implicitly included in the design matrix $\mathbf{G}$ ) indicates that the minimum resolvable length scale is not completely determined by the maximum frequency considered (related with a minimum wavelength), but also depends on station coverage. This arises generally if near-field data are used in the source inversion. The sensitivity of far-field waves can be intuitively characterized by their frequency-dependent wavelength. However, the near-field part of the wavefield is composed of evanescent waves that decay with distance from the fault, with a characteristic decay distance comparable to the wavelength of the slip source (e.g., Somala et al., 2014). Hence, each station has enhanced sensitivity in its vicinity. This example highlights the abstract nature of the coimage subspace: a truncated model, although robust, may display source patterns that are not directly amenable for physical interpretation. The enhanced sensitivity of the near-field observations is particularly visible when considering static displacement data, such as Interferometric Synthetic Aperture Radar data (e.g., Fukahata and Wright, 2008).

\section{Assessment of Inversion Methods Performance}

The inverted models agree with the target model after truncation generally well but not perfectly. The particular inversion methods also alter the resolvable (coimage) part of the solution. This could motivate a ranking of the inversion methods. However, further considerations are needed to generalize the present model comparison in this way. Indeed, the analysis is based on the GF matrix, which in practice is always affected by inaccuracies in the assumed fault-plane geometry, choice of station weights and frequency band of data filters, numerical issues in the evaluation of the GFs, and so on. All these factors affect the $\mathbf{G}$ matrix spectrum, and thus the comparison among different methods can be biased toward the one with the exact GFs. In other words, exact comparison can be made only if all the models use exactly the same GFs and the same inversion setup. One could also extend the comparison metric using multiple $\mathbf{G}$ matrices computed for a reasonable range of velocity models.

The comparison of SIV2a benchmark solutions presented here suggests that the linear methods perform better than the nonlinear ones. That might be true in this particular case where the fault-plane geometry and the GFs are almost exact. However, nonlinear methods can be potentially less sensitive to imperfections in the GFs, thus providing more robust (albeit less precise) solutions. They can also work with other objective functions than the L2 norm considered here, putting emphasis on different aspects of the wavefield and potentially leading to different solutions (Hartzell et al., 1991). Moreover, the nonlinear methods have the advantage of working with generally lower number of parameters, which enables effective uncertainty analysis.

Inversions that use only few parameters (represented here by model CedricT) are the most extreme cases, with just $\sim 20$ parameters. Their results compared with the target model after truncation at the twentieth singular value gives TMVR $=0.82$. Thus, from this perspective, the CedricT model is successful. However, such a severely truncated model does not even resemble the rupture propagation; hence, inferences on rupture processes cannot be readily extracted from it.

\section{CONCLUSIONS}

We introduced a new comparison technique based on an SVD of the design matrix of the continuum inverse problem. We propose to separate the coimage and null subspaces (representing resolved and unresolved features, respectively) by a selected cutoff singular value and compare different inverted models 
with the target (exact) model after projecting them on the coimage subspace (i.e., after truncation). This procedure effectively quantifies the ability of an inversion result to reproduce the resolvable features of the source.

As an example, we applied this technique to the SIV2a benchmark exercise within the SIV project (http://equake-rc. info/SIV/). The design matrix $\mathbf{G}$ of the SIV2a slip-inversion benchmark case has a smoothly decaying spectrum (see Fig. 1c) without a clear boundary between the coimage and the null space. The tests performed here (Picard plot and comparison of inversions from various authors) indicate that due to the use of imperfect GF the effective rank of $\mathbf{G}$ is limited to the subspace associated with singular values larger than one-tenth of the maximum singular value.

By applying our approach to the SIV2a benchmark results from various authors (see (E) Table S1), we show that the inferred source images are very similar to the target model after they all are truncated with cutoff at approximately one-tenth of the largest singular value. The models thus mainly differ in their respective contribution from the null-space, which is determined by the particular priors implied by their choices of regularization or parameterization and affected by the imperfections of the GFs. Although the truncated models capture the overall rupture propagation, their final slip distributions are biased significantly, showing distinct peaks below the stations lying above the rupture. This suggests that although the overall characteristics of the rupture propagation can be retrieved relatively well, the static slip distribution is not very well constrained. It also illustrates that the coimage subspace may contain abstract source patterns that are not directly amenable for interpretation.

Application of the comparison technique to inversions of a real event, with limited station coverage and imperfect knowledge of the fault geometry and GFs, would provide further insight into the quality of inferred source images. For such a comparative study, a well-studied event would have to be selected and all the modelers would have to consider the same basic setup (fault, mechanism, GFs, data processing, etc.) to carry out a fair comparison. $\mathbf{<}$

\section{ACKNOWLEDGMENTS}

We are grateful to all the participants in the Source Inversion Validation (SIV) benchmarks and to Martin Mai for his leadership in the SIV project, without whom this study would have been impossible. The article benefited from comments and suggestions by two anonymous reviewers. We acknowledge financial support from the Czech Science Foundation project 14-04372S, the Charles University project UNCE 204020/ 2012, the National Science Foundation (NSF) CAREER Award EAR-1151926, and the Southern California Earthquake Center (which is funded by NSF Cooperative Agreement EAR1033462 and U.S. Geological Survey Cooperative Agreement G12AC20038).

\section{REFERENCES}

Aki, K., and P. G. Richards (2002). Quantitative Seismology, University Science Books, Sausalito, California.

Asano, K., and T. Iwata (2011). Source rupture process of the 2007 Noto Hanto, Japan, earthquake estimated by the joint inversion of strong motion and GPS data, Bull. Seismol. Soc. Am. 101, $2467-2480$.

Bouchon, M. (1981). A simple method to calculate Green's functions for elastic layered media, Bull. Seismol. Soc. Am. 71, 959-971.

Clévédé, E., M.-P. Bouin, B. Bukchin, A. Mostinskiy, and G. Patau (2004). New constraints on the rupture process of the 1999 August 17 Izmit earthquake deduced from estimates of stress glut rate moments, Geophys. J. Int. 159, 931-942.

Coutant, O. (1989). Program of numerical simulation AXITRA, Research Report, Laboratoire de Géophysique Interne et Tectonophysique, Grenoble, France.

Di Stefano, R., C. Chiarabba, L. Chiaraluce, M. Cocco, P. De Gori, D. Piccinini, and L. Valoroso (2011). Fault zone properties affecting the rupture evolution of the $2009\left(M_{\mathrm{w}}\right.$ 6.1) L'Aquila earthquake (central Italy): Insights from seismic tomography, Geophys. Res. Lett. 38, L10310, doi: 10.1029/2011GL047365.

Duputel, Z., P. S. Agram, M. Simons, S. E. Minson, and J. L. Beck (2014). Accounting for prediction uncertainty when inferring subsurface fault slip, Geophys. J. Int. 197, no. 1, 464-482.

Fukahata, Y., and T. J. Wright (2008). A non-linear geodetic data inversion using ABIC for slip distribution on a fault with an unknown dip angle, Geophys. J. Int. 173, 353-364.

Gallovič, F., and J. Zahradník (2011). Toward understanding slip-inversion uncertainty and artifacts: 2. Singular value analysis, J. Geophys. Res. 116, no. B02309, doi: 10.1029/2010JB007814.

Gallovič, F., W. Imperatori, and P. M. Mai (2015). Effect of three-dimensional velocity heterogeneities and topography on slip inversions: Case study of the $M_{\mathrm{w}} 6.32009$ L'Aquila earthquake, J. Geophys. Res. 120, 428-449.

Hansen, P. Ch. (1990). The discrete Picard condition for discrete illposed problems, BIT 30, 658-672.

Hansen, P. Ch. (2010). Discrete Inverse Problems: Insight and Algorithms, Fundamentals of Algorithms Series , doi: 10.1137/ 1.9780898718836.

Hartzell, S., P. Liu, C. Mendoza, C. Ji, and K. M. Larson (2007). Stability and uncertainty of finite-fault slip inversions: Applications to the 2004 Parkfield, California, earthquake, Bull. Seismol. Soc. Am. 97, no. 6, 1911-1934.

Hartzell, S. H., G. S. Stewart, and C. Mendoza (1991). Comparison of L1 and L2 norms in a teleseismic waveform inversion for the slip history of the Loma Prieta, California, earthquake, Bull. Seismol. Soc. Am. 81, 1518-1539.

Hori, M. (2001). Inverse analysis method using spectral decomposition of Green's function, Geophys. J. Int. 147, 77-87.

Ide, S. (2007). Slip inversion, in Treatise on Geophysics, G. Schubert (Editor-in-Chief), H. Kanamori (Editor), Vol. 4, Elsevier, Amsterdam, The Netherlands, 193-223.

Jackson, D. D. (1972). Interpretation of inaccurate, insufficient, and inconsistent data, Geophys. J. 28, 97-109.

Menke, W. (2012). Geophysical Data Analysis: Discrete Inverse Theory, Third Ed., Academic Press, Boston, Massachusetts.

Olson, A. H., and R. J. Apsel (1982). Finite faults and inverse theory with applications to the 1979 Imperial Valley earthquake, Bull. Seismol. Soc. Am. 72, no. 6A, 1969-2001.

Razafindrakoto, H. N. T., and M. P. Mai (2014). Uncertainty in earthquake source imaging due to variations in source time function and earth structure, Bull. Seismol. Soc. Am. 104, 855-874.

Razafindrakoto, H. N. T., P. M. Mai, M. G. Genton, L. Zhang, and K. K. S. Thingbaijam (2015). An embedding method to quantify rupture model variability: Stability analysis and application to the $2011 \mathrm{M} 9$ Tohoku earthquake, Geophys. J. Int. 202, 17-40. 
Sekiguchi, H., K. Irikura, and T. Iwata (2000). Fault geometry at the rupture termination of the 1995 Hyogo-ken Nanbu earthquake, Bull. Seismol. Soc. Am. 90, 117-133.

Shao, G. F., and C. Ji (2012). What the exercise of the SPICE source inversion validation BlindTest 1 did not tell you, Geophys. J. Int. 189, 569-590.

Sokos, E., A. Kiratzi, F. Gallovič, J. Zahradník, A. Serpetsidaki, V. Plicka, J. Janský, J. Kostelecký, and G.-A. Tselentis (2015). Rupture process of the 2014 Cephalonia, Greece, earthquake doublet $\left(M_{\mathrm{w}} 6\right)$ as inferred from regional and local seismic data, Tectonophysics 656, 131-141.

Somala, S. N., J.-P. Ampuero, and N. Lapusta (2014). Resolution of rise time in earthquake slip inversions: Effect of station spacing and rupture velocity, Bull. Seismol. Soc. Am. 104, 2717-2734.

Tinti, E., E. Fukuyama, A. Piatanesi, and M. Cocco (2005). A kinematic source-time function compatible with earthquake dynamics, Bull. Seismol. Soc. Am. 95, 1211-1223.

Twardzik, C., R. Madariaga, S. Das, and S. Custódio (2012). Robust features of the source process for the 2004 Parkfield, California, earthquake from strong-motion seismograms, Geophys. J. Int. 191, 1245-1254.

Visser, R. (2004). A boundary element approach to acoustic radiation and source identification, Ph.D. Thesis, University of Twente, Enschede, the Netherlands.

Wiggins, R. (1972). The general linear inverse problem: Implications of surface waves and free oscillations on earth structure, Rev. Geophys. Space Phys. 10, 251-285.
Zahradník, J., and F. Gallovič (2010). Toward understanding slip-inversion uncertainty and artifacts, J. Geophys. Res. 115, no. B09310, doi: 10.1029/2010JB007814.

Zhang, L., P. M. Mai, K. K. S. Thingbaijam, H. N. Razafindrakoto, and M. G. Genton (2015). Comparing earthquake slip models with the spatial prediction comparison test, Geophys. J. Int. 200, 185-198.

F. Gallovič Charles University in Prague Faculty of Mathematics and Physics Department of Geophysics $V$ Holešovičkách 2 Prague 181 00, Czech Republic gallovic@karel.troja.mff.cuni.cz

J.-P. Ampuero Seismological Laboratory California Institute of Technology 1200 East California Boulevard Pasadena, California 91125 U.S.A.

Published Online 16 September 2015 